\section{The impact of off the visual axis retinoscopy on objective central refractive measurement in adult clinical practice: a prospective, randomized clinical study}

${ }^{1}$ Department of Ophthalmology, Charing Cross Hospital, London, UK

${ }^{2}$ Department of Ophthalmology, Moorfields Eye Hospital, London, UK

${ }^{3}$ Department of Epidemiology, National University of Singapore, Singapore

Correspondence:

$\checkmark$ Ferguson, Department of Ophthalmology, Charing Cross Hospital, Fulham Palace Road, London W6 8RF, UK.

E-mail:VFerguson@ hhnt.nhs.uk

Received: 17 May 2010 Accepted in revised form: 1 March 2011 Published online: 15 April 2011

\section{Abstract}

Purpose The objective of this study was to examine the effect of the off axis retinoscopy on objective central refractive measurement in adult clinical practice.

Methods In all, 40 subjects underwent undilated retinoscopy in a randomly selected eye both on and off the visual axis by a single masked examiner. Off axis retinoscopy was defined as retinoscopy performed with the testing eye of the examiner aligned with the contralateral (non-test) eye of the subject resulting in an off axis deviation in the nasal horizontal visual field. Retinoscopy was performed in negative cylinder only and spherocylindrical measurements were converted to power vectors for analysis. Paired $t$-test was used to assess differences in $M, J_{0}$ and $J_{45}$ power vectors including differences between mean aided and unaided LogMar acuities.

Results In all, 14 subjects were myopic $(\mathrm{SE} \leq-0.5 \mathrm{D}), 13$ subjects were emmetropic (SE between -0.49 and $1.0 \mathrm{D}$ ) and 13 subjects were hyperopic (SE $>1.0 \mathrm{D})$. Mean angle of deviation was $5.58^{\circ}$ in the nasal horizontal visual field. $\mathrm{J}_{0}$ showed a significant negative shift in those with myopia $(P<0.001)$ and emmetropia $(P=0.049)$ following off axis retinoscopy. No significant differences in $M$, $\mathrm{J}_{0}$ and $\mathrm{J}_{45}$ were found in the hyperopes. Mean aided LogMar acuities after on and off axis retinoscopy were both significantly better than mean unaided LogMar VA $(P<0.001$ in both cases).

Conclusion Small degrees of off axis retinoscopy encountered in everyday clinical practice can induce errors in objective central refractive measurement.

Eye (2011) 25, 888-892; doi:10.1038/eye.2011.79; published online 15 April 2011

Keywords: off axis; retinoscopy; refractive; error; power; vectors

\section{Introduction}

Retinoscopy is an important part of objective refractive measurement. Traditional teaching is that on the visual axis retinoscopy should be performed to obtain accurate measurements. ${ }^{1}$ The object of this study was to examine the effect of off the visual axis retinoscopy on objective central refractive measurement in routine adult clinical practice.

Materials and methods

Informed consent was obtained from all study subjects and the project was approved by the Charing Cross Research Ethics Committee. Inclusion criteria were adults with refractive errors and good red reflexes assessed with a pen torch. Exclusion criteria included children, 
non-English speakers and vulnerable groups. A total of 40 subjects, either patients or their accompanying relatives or hospital staff, were recruited from within the ophthalmology clinic. Each subject was provided with an information leaflet regarding the study and given sufficient time to give informed consent.

Each subject underwent undilated retinoscopy in a randomly selected eye by a single observer (LM) both on and off the visual axis in an examination room with uniform lighting. On the visual axis retinoscopy was performed first followed by off the visual axis retinoscopy with the examining observer leaving the room in between retinoscopy tests. This was performed to ensure that the examining observer was masked to the results of each retinoscopy trial. Steady fixation was achieved with the help of a distant target at $6 \mathrm{~m}$. The non-test eye was fogged with a plus lens to reduce involuntary accommodation. A second observer (ET), acting on the instructions of the examining observer, was responsible for the placement of lenses in the trial frame for the neutralization of the retinoscopic reflex. This was performed in a manner such that the examining observer was masked to the power of the selected lenses.

Retinoscopy was performed in negative cylinder only. Off axis retinoscopy was defined as retinoscopy performed with the testing eye of the observer aligned with the contralateral (non-test) eye of the subject resulting in an off axis deviation in the nasal horizontal field (Figure 1). A constant retinoscopic working distance of $66 \mathrm{~cm}$ was used. The visual acuity (unaided and pinhole), interpupillary distance and past ocular history were recorded in each case. The angle of deviation $(\alpha)$ was calculated with the formula $\tan \alpha=$ interpupillary distance in $\mathrm{mm}$ / working distance in $\mathrm{mm}$.

The spherical and cylindrical refractive errors obtained from retinoscopy were converted to power vectors according to the equations: ${ }^{2}$

$$
\begin{aligned}
\mathrm{M} & =\mathrm{S}(\text { sphere })+\mathrm{C}(\text { cylinder }) / 2 \\
\mathrm{~J}_{0} & =-(\mathrm{C} / 2) \cos (2 \theta) \\
\mathrm{J}_{45} & =-(\mathrm{C} / 2) \sin (2 \theta)
\end{aligned}
$$

Power vectors are the geometrical representation of spherocylindrical refractive errors, where $M$ is the spherical equivalent, $\mathrm{J}_{0}$ and $\mathrm{J}_{45}$ are the powers of the two Jackson crossed cylinders at axes $0^{\circ}$ and $45^{\circ}$, respectively, and $\theta$ is the cylindrical axis. Subjects were divided into refractive status according to spherical equivalent (SE) obtained following on axis retinoscopy: myopia (SE $\leq-0.5 \mathrm{D}$ ), emmetropia (SE between 0.49 and 1.0 D) and hyperopia (SE $>1.0 \mathrm{D}$ ). Paired $t$-test was used to assess the differences in $\mathrm{M}, \mathrm{J}_{0}$ and $\mathrm{J}_{45}$ obtained on and off the visual axis in each refractive group and also the differences between mean aided LogMar acuities following on and off axis retinoscopy and mean unaided LogMar acuities. Statistical analysis was performed with software SPSS (PASW Statistics 17, SPSS Inc., Chicago, IL, USA). Statistical significance was set at $P<0.05$.

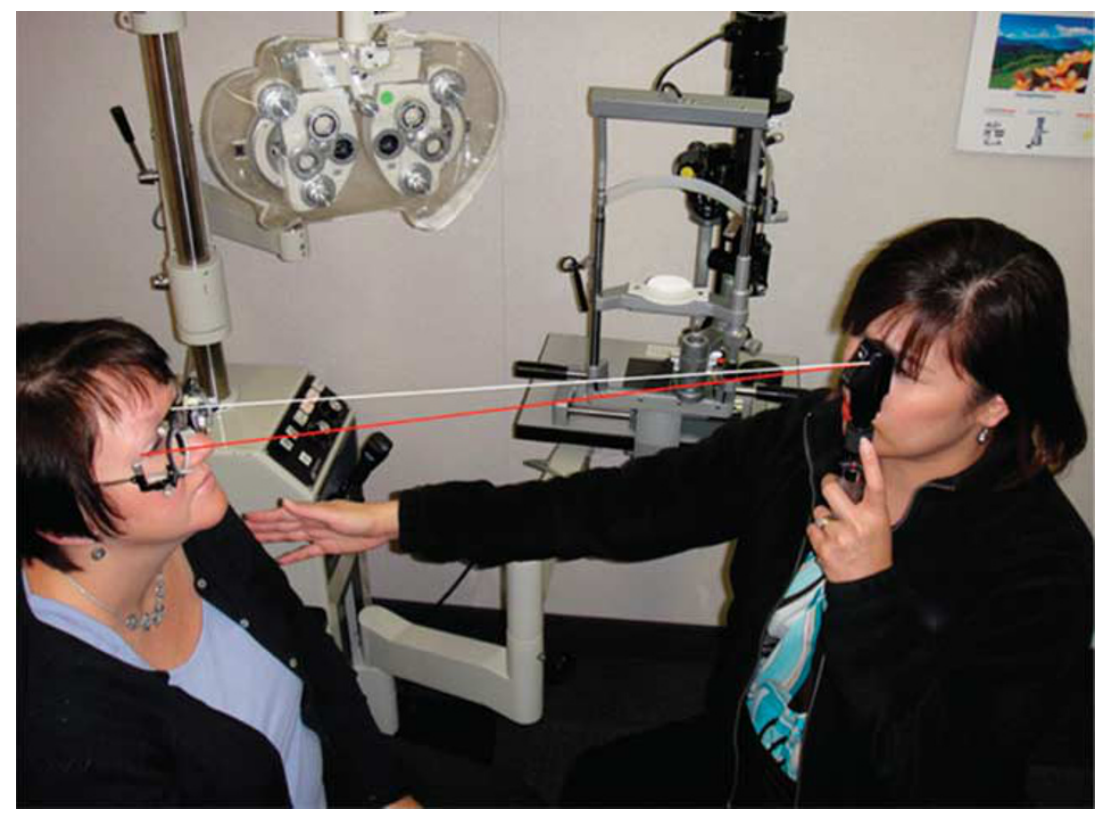

Figure 1 The examiner is using her left eye (which is aligned with the left eye of the patient, as shown by the white axis) to perform retinoscopy on the right eye of the patient (red axis) resulting in a small off the visual axis error in the nasal horizontal field. The left eye of the patient has been fogged with a plus lens. 


\section{Results}

A total of 40 subjects completed the study. A total of 20 right eyes and 20 left eyes underwent testing. There were 26 males and 14 females. In all, 14 subjects were myopic, 13 emmetropic and 13 hyperopic. Eight subjects had cataracts, four subjects were high myopes, one subject had narrow angle glaucoma, two subjects were pseudophakes, two subjects had age-related macular degeneration and one subject had pigment dispersion syndrome.

The mean age was 58.27 (range: $23-90$ ) years and the mean interpupillary distance was 64.67 (range: 55-72) $\mathrm{mm}$. Mean angle of deviation $(\alpha)$ was $5.58^{\circ}$ (range: 4.76-6.23) in the nasal horizontal visual field.

The mean aided LogMar visual acuities (VA) after on and off the visual axis retinoscopy were both significantly better than mean unaided LogMar VA $(0.11$ vs $0.50, P<0.001 ; 0.10$ vs $0.50, P<0.001$, respectively). There was no difference between mean aided LogMar VA after on and off the visual axis retinoscopy (0.11 vs 0.10 , $P=0.91)$. Mean astigmatic vector $\mathrm{J}_{0}$ showed a significant negative shift in those with myopia $(P<0.001)$ and emmetropia $(P=0.049)$ following off axis retinoscopy. No significant differences in $\mathrm{M}, \mathrm{J}_{0}$ and $\mathrm{J}_{45}$ were found in those with hyperopia. The distributions of $\mathrm{M}, \mathrm{J}_{0}$ and $\mathrm{J}_{45}$ in each refractive group following on and off axis retinoscopy are shown in Table 1.

\section{Discussion}

In practical terms, retinoscopy is frequently performed slightly off the visual axis and there are situations in which on the visual axis retinoscopy is difficult or even impossible. These include retinoscopy in uncooperative children or when the subject has been anaesthetized. There has been little research on the effects of off the visual axis retinoscopy on objective central refractive measurement in adults in the clinical setting in recent years.
Rempt et $a l^{3}$ was the first to perform eccentric retinoscopy and introduced the skiagram to categorize different peripheral refractive error patterns. Ferree $e^{2} a l^{4}$ in 1931 measured peripheral refraction with the Zeiss parallax refractometer (Carl Zeiss, Jena, Germany), which is an accurate but cumbersome device. Later studies either used a theoretical model ${ }^{5}$ or measured peripheral refraction at large angles of eccentricity (from $20^{\circ}$ ) that are unlikely to be encountered in everyday practice. , $, 5,6$ A number of these studies also conducted eccentric retinoscopy under cycloplegia ${ }^{3,6,7}$ making comparisons with routine adult clinical examination (usually undilated retinoscopy) difficult. These studies were aimed at determining peripheral refraction and their findings do not actually address the effect of off the visual axis testing on the accuracy of objective central refractive measurement.

Jackson $e t a l^{8}$ examined off the visual axis retinoscopy in eight adults (spherical equivalent $< \pm 2.5 \mathrm{D}$ ) who had undergone cycloplegia. Degrees of eccentricity of up to $20^{\circ}$ along the temporal horizontal field were examined and he found a myopic shift of $5 \%$ in spherical equivalent and an increase in cylindrical power of $3 \%$ for every degree of eccentricity. As eccentric retinoscopy is thought to produce a hyperopic refractive shift ${ }^{9,10}$ because of the peripheral corneal flattening and testing along the shorter, off the visual axis, axial length, ${ }^{11}$ he cited previous work on intraocular lenses showing the induction of myopic and astigmatic errors when light strikes a lens obliquely ${ }^{12}$ to support his findings. A subsequent study examining refraction across the central $10^{\circ}$ of the horizontal visual field in dilated subjects with a Hartmann-Shack wavefront sensor found a maximum change of $0.5 \mathrm{D}$ in mean sphere, ${ }^{13}$ supporting Jackson's findings of retinoscopic changes occurring within a few degrees of central fixation.

There has been recent interest in the role peripheral refraction on the development of central refractive error.

Table $1 \mathrm{M}, \mathrm{J}_{0}$ and $\mathrm{J}_{45}$ for the different refractive groups following on and off the visual axis retinoscopy

\begin{tabular}{|c|c|c|c|c|}
\hline Refractive status & Power vectors & $\begin{array}{c}\text { On the visual axis } \\
\text { Mean }(S D)\end{array}$ & $\begin{array}{c}\text { Off the visual axis } \\
\text { Mean (SD) }\end{array}$ & $\begin{array}{c}\text { P-value } \\
\text { (paired t-test) }\end{array}$ \\
\hline Myopia (SE $\leq-0.50 \mathrm{D})$ & $\begin{array}{l}\mathrm{M} \\
\mathrm{J}_{0} \\
\mathrm{~J}_{45}\end{array}$ & $\begin{array}{r}-3.34(2.76) \\
0.05(0.16) \\
0.06(0.17)\end{array}$ & $\begin{array}{l}-3.28(2.73) \\
-0.23(0.30) \\
-0.03(0.35)\end{array}$ & $\begin{array}{c}0.068 \\
<0.001^{\mathrm{a}} \\
0.36\end{array}$ \\
\hline Emmetropia (SE between -0.49 and $1.0 \mathrm{D}$ ) & $\begin{array}{l}\mathrm{M} \\
\mathrm{J}_{0} \\
\mathrm{~J}_{45}\end{array}$ & $\begin{array}{r}0.17(0.37) \\
-0.16(0.57) \\
-0.09(0.15)\end{array}$ & $\begin{array}{l}-0.17(0.64) \\
-0.40(0.47) \\
-0.18(0.47)\end{array}$ & $\begin{array}{l}0.08 \\
0.049^{\mathrm{a}} \\
0.51\end{array}$ \\
\hline Hyperopia (SE > 1.0 D) & $\begin{array}{l}\mathrm{M} \\
\mathrm{J}_{0} \\
\mathrm{~J}_{45}\end{array}$ & $\begin{array}{r}2.34(1.32) \\
-0.30(0.34) \\
0.04(0.23)\end{array}$ & $\begin{array}{r}2.45(1.43) \\
-0.39(0.33) \\
-0.06(0.34)\end{array}$ & $\begin{array}{l}0.50 \\
0.16 \\
0.40\end{array}$ \\
\hline
\end{tabular}

${ }^{a}$ Denotes statistical significance. 
Hoogerheide et $a^{14}$ in 1971 was the first to observe relative peripheral hyperopia in pilots who later developed myopia. Subsequent studies by Mutti et $a l^{15}$ and Sng et $a^{16}$ have also shown relative peripheral hyperopia in myopic individuals and relative peripheral myopia in emmetropic and hyperopic individuals.

Peripheral refraction profiles may differ in the horizontal and vertical visual fields. Atchison $e t a l^{17}$ found peripheral myopic shifts in emmetropes and hyperopic shifts in myopes for $\mathrm{M}$ along the horizontal visual field using a Shin Nippon SRW-5000 autorefractor (Ajinomoto Trading Inc., Tokyo, Japan) in a cohort of 116 undilated subjects. Along the vertical visual field, however, both groups showed myopic shifts in M. With regards $\mathrm{J}_{0}$, positive refraction shifts occurred along the vertical visual field and negative refraction shifts occurred along the horizontal visual field. ${ }^{17}$ A linear relationship between $\mathrm{J}_{45}$ and visual field angle was found along both horizontal and vertical visual fields with positive refraction shifts occurring along the superior and nasal visual fields and negative refraction shifts occurring along the inferior and temporal visual fields. ${ }^{17}$

As cycloplegia is not routinely induced in adults undergoing refraction, it was our aim to mimic clinical practice by examining the effect of off the visual axis retinoscopy without cycloplegia. We defined off the visual axis retinoscopy as retinoscopy performed with the testing eye of the examiner aligned with the contralateral (non-test) eye of the subject. In practical terms, this occurs when a retinoscopist uses the same eye to examine both eyes of the subject without changing seating position and is probably the most commonly encountered 'bad habit' in clinical practice. This results in a mean angle of eccentricity of $5.58^{\circ}$ in the nasal direction along the horizontal visual field, which although small, is much more likely to be encountered than the larger angles of eccentricity assessed in other studies.

We found significant changes in $\mathrm{J}_{0}$ following off axis retinoscopy in subjects with myopia and emmetropia. These findings are supported by the work of Atchison et $a l_{,}{ }^{17}$ in which negative shifts in $\mathrm{J}_{0}$ were found along the horizontal visual field up to $35^{\circ}$ of eccentricity in myopic and emmetropic subjects. We did not find any change in $\mathrm{M}$ and $\mathrm{J}_{45}$ perhaps because of the small angle of eccentricity tested or the lower resolution of the retinoscopy technique compared with that of an autorefractor. In addition, $\mathrm{J}_{45}$ changes in the periphery of emmetropes have been shown to be much smaller than $\mathrm{J}_{0}$ changes $^{18}$ making them harder to measure.

We did not find any significant change in $\mathrm{M}, \mathrm{J}_{0}$ and $\mathrm{J}_{45}$ following off axis retinoscopy in our hyperopic subjects. There is little published data in this area and a previous study found a myopic shift in $\mathrm{M}$ into the nasal field in four of six hyperopic subjects with an increase in $\mathrm{J}_{0}$ in the periphery. ${ }^{18}$

The order of retinoscopy in each subject was not randomized, but we do not feel that this introduced any significant order effect. We also did not measure pupil size and were unable to correlate pupil size with the error induced. However, all subjects had retinoscopy performed under uniform lighting conditions and we did not observe any pupils significantly outside the $2-4 \mathrm{~mm}$ range.

In conclusion, our study confirms the traditionally held belief that off the visual axis retinoscopy induces errors in objective central refractive measurement. Although these small errors are usually eliminated following subjective refraction, it is still good clinical practice to perform retinoscopy as close to the visual axis whenever possible.

\section{Summary}

What was known before

- Traditional teaching is that retinoscopy should be performed on the visual axis for accurate results. However, there are no recent studies that mimic actual adult clinical practice to verify this assumption. Previous studies have either used autorefractors, cycloplegia or tested angles of eccentricity that are too large to occur in real life.

\section{What this study adds}

- This is the first study to assess the effect of off axis retinoscopy on objective central refractive assessment in undilated adults. It shows that the most common error committed by retinoscopists (ie, using the same eye to perform retinoscopy on both subject's eyes from the same seated position) results in a small angle of eccentricity, which still leads to a significant error in objective central refractive measurement.

\section{Conflict of interest}

The authors declare no conflict of interest.

\section{References}

1 Michaels DD. Visual Optics And Refraction A Clinical Approach, 3rd ed. Mosby: St Louis, 1985; 305.

2 Thibos LN, Wheeler W, Horner D. Power vectors: an application of Fourier analysis to the description and statistical analysis of refractive error. Optom Vis Sci 1997; 74: 367-375.

3 Rempt F, Hoogerheide J, Hoogenboom WP. Peripheral retinoscopy and the skiagram. Ophthalmologica 1971; 162: $1-10$

4 Ferree CE, Rand G, Hardy C. Refraction for the peripheral field of vision. Arch Ophthlmol 1931; 5: 717-731.

5 Lotmar W, Lotmar T. Peripheral astigmatism in the human eye: experimental data and theoretical model predictions. J Opt Soc Am 1974; 64: 510-513. 
6 Millidot M, Lamont A. Refraction of the periphery of the eye. J Opt Soc Am 1974; 64: 110-111.

7 Rempt F, Hoogerheide J, Hoogenboom WP. Influence of correction of peripheral refractive errors on peripheral static vision. Ophthalmologica 1976; 173: 128-135.

8 Jackson DW, Paysse EA, Wilhelmus KR, Hussein MA, Rosby G, Coats DK. The effect of off the visual axis retinoscopy on objective refractive measurement. Am J Ophthalmol 2004; 137: 1101-1114.

9 Johnson CA, Leibowitz HW. Practice, refractive error and feedback as factors influencing peripheral motion thresholds. Percept Psychophys 1974; 15: 276-280.

10 Leibowitz HW, Johnson CA, Isabelle E. Peripheral motion detection and refractive error. Science 1972; 177: 1207-1208.

11 Berges O, Puech M, Assouline M, Letenneur L, Gastellu-Etchegorry M. B mode guided vector A mode versus A mode biometry to determine axial length and intraocular lens power. J Cataract Refract Surg 1998; 24: 529-535.
12 Erickson P. Effects of intraocular lens position errors on postoperative refractive error. J Cataract Refract Surg 1990; 16: 305-311.

13 Atchison DA, Lucas SD, Ashman R, Huynh MA, Schilt DW, Ngo PQ. Refraction and aberration across the horizontal central $10^{\circ}$ of the visual field. Optom Vis Sci 2006; 83: 213-221.

14 Hoogerheide J, Rempt F, Hoogenboom WPH. Acquired myopia in young pilots. Ophthalmologica 1971; 163: 209-215.

15 Mutti DO, Sholtz RI, Friedman NE, Zadnik K. Peripheral refraction and ocular shape in children. Invest Ophthalmol Vis Sci 2000; 41: 1022-1030.

16 Sng C, Lin X, Gazzard G, Chang B, Dirani M, Chia A et al. Peripheral refraction and refractive error in Singapore Chinese children. Invest Ophthalmol Vis Sci 2011; 52: 1181-1190.

17 Atchison DA, Pritchard N, Schmid KL. Peripheral refraction along the horizontal and vertical visual fields in myopia. Vision Res 2006; 46: 1450-1458.

18 Ma L, Atchison DA, Charman WN. Off axis refraction and aberrations following conventional laser in situ keratomileusis. J Cataract Refract Surg 2005; 31: 489-498. 